PROCEEDINGS OF THE

AMERICAN MATHEMATICAL SOCIETY

Volume 134, Number 6, Pages 1835-1847

S 0002-9939(05)08306-1

Article electronically published on December 5, 2005

\title{
QUARTER-STRATIFIABILITY IN ORDERED SPACES
}

\author{
HAROLD R. BENNETT AND DAVID J. LUTZER
}

(Communicated by Alexander N. Dranishnikov)

\begin{abstract}
In this paper we study Banakh's quarter-stratifiability among generalized ordered (GO)-spaces. All quarter-stratifiable GO-spaces have a $\sigma$ closed-discrete dense set and therefore are perfect, and have a $G_{\delta}$-diagonal. We characterize quarter-stratifiability among GO-spaces and show that, unlike the situation in general topological spaces, quarter-stratifiability is a hereditary property in GO-spaces. We give examples showing that a separable perfect GO-space with a $G_{\delta}$-diagonal can fail to be quarter-stratifiable and that any GO-space constructed on a Q-set in the real line must be quarter-stratifiable.
\end{abstract}

\section{INTRODUCTION}

In [8, W. Rudin proved that if $X$ is a metric space, then for every topological space $Y$, every separately continuous real-valued function on $X \times Y$ is of the first Baire class. In [5] and [7, Kuratowski and Montgomery proved that for metric spaces $X, Y$ and $Z$, a function $f: X \times Y \rightarrow Z$ is Borel measurable of countable class $\alpha+1$, provided $f$ is continuous in one of its variables and of Borel class $\alpha$ in the other variable. In [2], T.O. Banakh introduced a broad generalization of metric spaces that he called "metrically quarter-stratifiable spaces" and proved the above theorems of Rudin, Kuratowski, and Montgomery under the assumption that $X$ belongs to this new class.

According to Banakh [2], a topological space $(X, \tau)$ is quarter-stratifiable if there is a function $g$ (called a quarter-stratification of $X$ ) from $\{1,2,3, \cdots\} \times X$ into $\tau$ such that

a) for each $n \geq 1$, the collection $\{g(n, x): x \in X\}$ covers $X$;

b) if $y \in g\left(n, x_{n}\right)$ for each $n$, then the sequence $\left\langle x_{n}\right\rangle$ converges to $y$.

If there is a metrizable topology $\mu \subseteq \tau$ on $X$ such that $g(n, x) \in \mu$ for each $n$ and each $x$, then $(X, \tau)$ is metrically quarter-stratifiable and the function $g$ is said to be a metric quarter-stratification for $(X, \tau)$.

It is important to note that, in the definition of quarter-stratifiability, $x$ is not required to be a point of $g(n, x)$, and this distinguishes quarter-stratifiable spaces from most other types of generalized metric spaces in use today. For example, in the definition of quarter-stratifiable spaces, replacing the assertion that each $\{g(n, x): x \in X\}$ covers $X$ by the requirement that $x \in g(n, x)$ for each $n$ and each $x$ characterizes Creede's semi-stratifiable spaces [3]. To see how major a change this is, note that the familiar Sorgenfrey line $\mathbb{S}$ is quarter-stratifiable (using the

Received by the editors January 12, 2005 .

2000 Mathematics Subject Classification. Primary 54F05; Secondary 54E20, 54 H05.

(C)2005 American Mathematical Society Reverts to public domain 28 years from publication 
function $g(n, x)=\left(x-\frac{1}{n}, x-\frac{1}{2 n}\right)$ for each rational $x$ and $g(n, x)=\emptyset$ for each irrational $x$ ) but is not semi-stratifiable because, among GO-spaces, the four properties of metrizability, developability, semi-metrizability, and semi-stratifiability are mutually equivalent [6].

The purpose of this paper is to investigate the theory of quarter-stratifiability in the class of generalized ordered spaces. Recall that a generalized ordered space (GO-space) is a triple $(X,<, \tau)$, where $<$ is a linear ordering of the set $X$ and $\tau$ is a $T_{1}$ topology on $X$ that has a base of open sets whose members are order-convex. The open-interval topology $\lambda$ of the order $<$ always has $\lambda \subseteq \tau$. If $\lambda=\tau$, then $X$ is a linearly ordered topological space (LOTS). As proved by E. Čech, GO-spaces are exactly those topological spaces that embed (topologically) in some LOTS.

The main results in our paper are: (i) a list of properties of every quarterstratifiable GO-space (see Proposition 2.2) that includes first-countability, hereditarily paracompactness, having a $G_{\delta}$-diagonal, and having a $\sigma$-closed-discrete dense set, so that every quarter-stratifiable GO-space is perfect and there are no quarterstratifiable Souslin spaces; (ii) a characterization of quarter-stratifiable GO-spaces (Theorem 3.1) in terms of the special structure of the space's right- and left-looking points; (iii) Theorem 4.1 showing that, unlike the situation in general quarterstratifiable spaces, the class of quarter-stratifiable GO-spaces is a hereditary class; and (iv) a family of examples of GO-spaces constructed on the usual space of real numbers illustrating earlier results of the paper.

We reserve the symbols $\mathbb{Q}, \mathbb{P}$, and $\mathbb{R}$ for the sets of rational, irrational, and real numbers, respectively, with their usual orders. The authors would like to thank the referee for comments that substantially improved the first version of this paper.

\section{Properties of QUARTER-STRATifiable ORDERED SPACES}

Lemma 2.1. Suppose $g(n, x)$ is a quarter-stratification function for any $T_{2}$-space $X$. Then each set

$$
F(n)=\{x \in X: x \in g(n, z) \Rightarrow z=x\}
$$

is a closed discrete subset of $X$.

Proof. Let $p \in X$. Choose any point $z \in X$ with $p \in g(n, z)$. Then $q \in g(n, z) \cap F(n)$ forces $q=z$ so that $g(n, z) \cap F(n)=\{z\}$. Hence $p$ is not a limit point of $F(n)$. But then $F(n)$ is closed and discrete.

Recall that a topological space $X$ is perfect if each closed subset of $X$ is a $G_{\delta^{-}}$ subset of $X$.

Proposition 2.2. Let $X$ be a quarter-stratifiable GO-space. Then:

a) $X$ has a $G_{\delta}$-diagonal;

b) the density of $X$ equals the Lindelöf degree of $X$;

c) $X$ is first countable and hereditarily paracompact;

d) $X$ is metrically quarter-stratifiable;

e) $X$ has a $\sigma$-closed-discrete dense subset and therefore is perfect.

Proof. In 2], Banakh proved (a) for any quarter-stratifiable $T_{2}$-space and showed that density $\leq$ Lindelöf degree in any quarter-stratifiable space. It is well known 6] that Lindelöf degree $\leq$ density for any GO-space, so that (b) is established. It is known [6] that any GO-space with a $G_{\delta}$-diagonal is hereditarily paracompact. 
Furthermore, the $G_{\delta}$-diagonal makes each point of $X$ a $G_{\delta}$-set in $X$ so that $X$ is firstcountable. Thus, (c) holds. Finally, Banakh proved in his Theorem 2.3 that any paracompact Hausdorff quarter-stratifiable space is metrically quarter-stratifiable, so that (d) holds.

To prove assertion (e), we use (a) together with a result of Przymusinski [1] to find a metrizable topology $\mu$ such that $\mu \subseteq \tau$ and such that $(X, \mu,<)$ is also a GO-space. Let $D \subseteq X$ be a dense subset of $(X, \mu)$ that is $\sigma$-closed-discrete in $(X, \mu)$. Let $I$ be the set of all isolated points of $(X, \tau)$. Once we show that $I$ is an $F_{\sigma}$-subset of $(X, \tau)$, it will follow that $D \cup I$ is the required dense $\sigma$-closed-discrete subset of $(X, \tau)$. We will complete the proof by applying Faber's result [4 that any GO-space with a $\sigma$-closed-discrete dense set must be perfect.

Suppose $g(n, x)$ is a quarter-stratification function for $X$. Let $I(n)=\{y \in I$ : $y \in g(n, z) \Rightarrow z=y\}$. Then $I(n) \subseteq F(n)$, where $F(n)$ is the set defined in Lemma 2.1. so $I(n)$ is closed and discrete. It remains to show that $I \subseteq \bigcup\{I(n): n \geq 1\}$, the other containment being obvious. Let $p \in I$ and suppose that for each $n \geq 1$ there is a point $z(n) \neq p$ with $p \in g(n, z(n))$. But then $\langle z(n)\rangle$ must converge to $p$, and that is impossible because $p$ is isolated.

Remark 2.3. In the light of Proposition 2.2(a), each quarter-stratifiable LOTS is metrizable. However, as the Sorgenfrey line shows, a GO space can be quarterstratifiable and non-metrizable.

The fact that quarter-stratifiable GO-spaces must be perfect contrasts sharply with the behavior of arbitrary quarter-stratifiable spaces. Banakh [2] gave an example of a separable zero-dimensional metrically quarter-stratifiable Tychonoff space that is not perfect. The referee pointed out that part (e) of Proposition 2.2 raises a more general question:

Question 2.4. Is it true that each paracompact quarter-stratifiable $T_{2}$-space (i.e., not necessarily a GO-space) contains a dense subset that is $\sigma$-closed-discrete?

\section{Which GO SPACES ARE QUARTER-STRATIFIABLE?}

In this section we characterize those GO-spaces that are quarter-stratifiable in terms of certain special subsets $R, E, I$ and $L$ of any GO-space. For any GO-space $(X,<, \tau)$, let $I(X)$ be the set of all isolated points of $(X, \tau)$. Define $R(X)=$ $\{x \in X-I(X):[x, \rightarrow) \in \tau\}$ and $L(X)=\{x \in X-I(X):(\leftarrow, x] \in \tau\}$. Let $E(X)=X-(I(X) \cup R(X) \cup L(X))$. In addition, for any subset $S$ of $X$, let $S^{\text {isol }}$ denote the set of relatively isolated points of $S$, i.e. points of $S$ that are not limit points of $S$.

Warning: These notations are not completely standard in GO-space theory; some authors define $R(X)$ and $L(X)$ in such a way that each isolated point of $X$ is in $R(X) \cap L(X)$, but in this paper we will need the sets $R(X), E(X), I(X)$, and $L(X)$ to be pairwise disjoint.

The referee pointed out that assertions (c) and (d) in the following theorem are equivalent to (a) and (b).

Theorem 3.1. Let $(X, \tau,<)$ be a perfect $G O$-space with a $G_{\delta}$-diagonal. Then the following are equivalent:

(a) $X$ is quarter-stratifiable. 
(b) With $R(X)$ and $L(X)$ as defined above, there are sets $R(n), L(n)$ with

$(\mathrm{b}-1) \quad R(n) \subseteq R(n+1), L(n) \subseteq L(n+1), R(X)=\bigcup\{R(n): n \geq 1\}$ and $L(X)=\bigcup\{L(n): n \geq 1\}$

(b-2) $\mathrm{cl}_{X}(R(n)) \cap \mathrm{cl}_{X}(L(n)) \subseteq E(X) \cup R(n)^{\text {isol }} \cup L(n)^{\text {isol }}$ for each $n \geq 1$;

(b-3) if $x \in E(X)$, then for each $n \geq 1$ there is an open convex neighborhood $M(n, x)$ of $x$ with the property that $a<b$ for any $a \in M(n, x) \cap R(n)$ and any $b \in M(n, x) \cap L(n)$.

(c) There is a $\sigma$-closed-discrete subset $Q$ of $X$ such that in the subspace $X-Q$ there are disjoint relative- $F_{\sigma}$-sets $A$ and $B$ with $R(X)-Q \subseteq A$ and $L(X)-$ $Q \subseteq B$.

(d) There is some subset $Q \subseteq X$ such that both $R(X) \cap Q$ and $L(X) \cap Q$ are $\sigma$ closed discrete, and in the subspace $X-Q$ there are disjoint relative- $F_{\sigma}$-sets $A$ and $B$ with $R(X)-Q \subseteq A$ and $L(X)-Q \subseteq B$.

Obviously (c) implies (d). The remainder of this section presents a sequence of lemmas that, together, prove the rest of Theorem 3.1 .

Lemma 3.2. Suppose $g(n, x)$ is a quarter-stratification function for the GO-space $X$. Then

(i) for each $x \in R(X)$ there is an integer $N(x) \geq 1$ such that if $x \in g(k, y)$ and $k \geq N(x)$, then $x \leq y$. In addition, if $R(n)=\{x \in R(X): N(x) \leq n\}$, then $R(n) \subseteq R(n+1)$ and $R(X)=\bigcup\{R(n): n \geq 1\}$;

(ii) for each $x \in L(X)$ there is an integer $N(x) \geq 1$ such that if $x \in g(k, y)$ and $k \geq N(x)$, then $y \leq x$. In addition, if $L(n)=\{x \in L(X): N(x) \leq n\}$, then $L(n) \subseteq L(n+1)$ and $L=\bigcup\{L(n): n \geq 1\}$;

(iii) $\operatorname{cl}_{X}(R(n)) \cap \mathrm{cl}_{X}(L(n)) \subseteq E(X) \cup R(n)^{i s o l} \cup L(n)^{i s o l}$;

(iv) for each $x \in E(X)$ there is a convex open set $M(n, x)$ with the property that if $a \in R(n) \cap M(n, x)$ and $b \in L(n) \cap M(n, x)$, then $a<b$.

Therefore, in Theorem 3.1. (a) $\Rightarrow$ (b).

Proof of Lemma 3.2. Consider the first assertion. For a contradiction, suppose no $N(x)$ exists. Then there is a strictly increasing sequence $n_{i}$ and points $t_{n_{i}}<x$ with $x \in g\left(n_{i}, t_{n_{i}}\right)$. For each $n \geq 1$ with $n \notin\left\{n_{i}: i \geq 1\right\}$, let $t_{n}$ be any point of $X$ with $x \in g\left(n, t_{n}\right)$. Then $\left\langle t_{n}\right\rangle$ must converge to $x$ because $g$ is a quarter-stratification, and that is impossible, because no term $t_{n_{i}}$ is in the open set $[x, \rightarrow)$. Hence $N(x)$ exists and assertion (i) holds. Assertion (ii) is proved analogously.

To prove assertion (iii), suppose that $p$ is a limit point of both $R(n)$ and $L(n)$ and that $p \notin E(X)$. Because no point of $I(X)$ can be a limit point of any set, we know that $p \in R(X)$ or $p \in L(X)$. Consider the first case, the other being analogous. Choose any $t \in X$ with $p \in g(n, t)$. Because $g(n, t) \cap[p, \rightarrow)$ is a neighborhood of $p$ there is some $x \in R(n) \cap[p, \rightarrow) \cap g(n, t)$ with $p<x$. Because $g(n, t) \cap[p, x)$ is a neighborhood of $p$ there is some $y \in g(n, t) \cap[p, x) \cap L(n)$. Then $y<x$. However, $y \in L(n)$ and $y \in g(n, t)$ imply $t \leq y$ while $x \in R(n) \cap g(n, t)$ forces $x \leq t$ so that we have $t \leq y<x \leq t$, and that is impossible. Hence (iii) holds.

To verify assertion (iv), suppose $x \in E(X)$ and choose any $g(n, t)$ with $x \in$ $g(n, t)$. As in the proof of assertion (iii), if $a \in R(n) \cap g(n, t)$ and $b \in L(n) \cap g(n, t)$, then $a<b$. We define $M(n, x)$ to be the convex component of $g(n, t)$ that contains $x$.

Lemma 3.3. Suppose $X$ is a perfect $G O$-space with a $G_{\delta}$-diagonal. Then in Theorem 3.1, (b) $\Rightarrow(\mathrm{c})$. 
Proof. In this proof, all closures are taken in the space $X$. Given sets $R(n)$ and $L(n)$ as in part (b) of the statement of Theorem 3.1 define $Q=\bigcup\{\operatorname{cl}(R(n)) \cap \operatorname{cl}(L(n))$ : $n \geq 1\}$.

Claim 1. The set $Q$ is $\sigma$-closed-discrete. Because $X$ is perfect, it is enough to show that each set $\operatorname{cl}(R(n)) \cap \operatorname{cl}(L(n))$ is the union of finitely many subsets, each being discrete in itself. To that end, fix $n$ and let

$$
\begin{aligned}
& D_{1}=\{p \in R(n) \cap \operatorname{cl}(L(n)): p \text { is relatively isolated in } R(n)\} ; \\
& D_{2}=\{p \in L(n) \cap \operatorname{cl}(R(n)): p \text { is relatively isolated in } L(n)\} ; \\
& D_{3}=\{p: p \text { is a limit point of both } R(n) \text { and } L(n)\} .
\end{aligned}
$$

Clearly $\operatorname{cl}(R(n)) \cap \operatorname{cl}(L(n))=D_{1} \cup D_{2} \cup D_{3}$. The set of relatively isolated points of $R(n)$ (respectively $L(n)$ ) is relatively discrete. Hence so are $D_{1}$ and $D_{2}$. For contradiction, suppose that $D_{3}$ contains a limit point $p$ of itself. Choose distinct points $q_{j} \in D_{3}$ with $p=\lim \left\langle q_{j}\right\rangle$. Without loss of generality, we may assume that $q_{1}<q_{2}<\cdots$. From (b-2) of Theorem 3.1, we know that $p \in E$. Let $M=M(p, n)$ be the open neighborhood of $p$ described in (b-3) of Theorem 3.1. Then $a<b$ whenever $a \in M \cap R(n)$ and $b \in M \cap L(n)$. Choose $i<j$ with $q_{i}, q_{j} \in M$. Because $q_{i}, q_{j} \in D_{3}$, there are open, convex, disjoint neighborhoods $J_{1}, J_{2}$ of $q_{i}$ and $q_{j}$, respectively, with $J_{i} \subseteq M$ and with the property that $x<y$ whenever $x \in J_{1}$ and $y \in J_{2}$. Because $q_{i} \in D_{3}, q_{i}$ is a limit point of $L(n)$ so that there is some point $x \in L(n) \cap J_{1}$. Similarly, there is some point $y \in J_{2} \cap R(n)$. But then $x \in M \cap L(n)$ and $y \in M \cap R(n)$ even though $x<y$, which is impossible. Therefore, $D_{3}$ contains no limit point of itself.

Claim 2. The sets $R(X)-Q$ and $L(X)-Q$ are contained in disjoint, relatively $F_{\sigma^{-}}$ subsets of $X-Q$. Let $A=(\bigcup\{\operatorname{cl}(R(n)): n \geq 1\}) \cap(X-Q)$ and $B=(\bigcup\{\operatorname{cl}(L(n))$ : $n \geq 1\}) \cap(X-Q)$. Then $A, B$ are relative $F_{\sigma}$-subsets of $X-Q$ and $R(X)-Q \subseteq A$ while $L(X)-Q \subseteq B$. It remains to show that $A \cap B=\emptyset$. Fix any $m, n \geq 1$. If $m \leq n$, then $\operatorname{cl}(R(n)) \cap \operatorname{cl}(L(m)) \subseteq \operatorname{cl}(R(n)) \cap \operatorname{cl}(L(n)) \subseteq Q$, and if $n \leq m$, then $\operatorname{cl}(R(m)) \cap \operatorname{cl}(L(n)) \subseteq \operatorname{cl}(R(m)) \cap \operatorname{cl}(L(m)) \subseteq Q$. Hence $A \cap B=\emptyset$.

Lemma 3.4. Suppose that $X$ is a perfect $G O$-space with a $G_{\delta}$-diagonal and that $X$ satisfies assertion (d) of Theorem 3.1. Then $X$ also satisfies assertion (b) of that theorem.

Proof. In this proof, $\operatorname{cl}(S)$ denotes the closure of a set $S$ in the space $X$. We have a set $Q \subseteq X$ such that $Q \cap(R(X) \cup L(X))$ is $\sigma$-closed-discrete in $X$ and two disjoint relative $F_{\sigma}$-subsets $A, B \subseteq X-Q$ with $R(X)-Q \subseteq A$ and $L(X)-Q \subseteq B$. Write $A=\bigcup\{A(n): n \geq 1\}$ and $B=\bigcup\{B(n): n \geq 1\}$, where $A(n) \subseteq A(n+1), B(n) \subseteq$ $B(n+1)$ and where each of the sets $A(n), B(n)$ is relatively closed in the subspace $X-Q$. Then for any $m, n \geq 1, \operatorname{cl}(A(m)) \cap \operatorname{cl}(B(n)) \subseteq Q$.

Because $X$ is perfect, the set $\operatorname{cl}(B(n))$ is a $G_{\delta}$-subset of $X$. Hence the set $\operatorname{cl}(A(n))-\operatorname{cl}(B(n))$ is an $F_{\sigma}$-subset of $X$. Write

$$
\operatorname{cl}(A(n))-\operatorname{cl}(B(n))=\bigcup\{H(n, j): n \geq 1\},
$$

where $H(n, 1) \subseteq H(n, 2) \subseteq \cdots$ are closed subsets of $X$. Similarly we write $\operatorname{cl}(B(n))-\operatorname{cl}(A(n))$ as the increasing union $\bigcup\{K(n, j): j \geq 1\}$ of closed subsets of $K(n, j) \subseteq X$. 
Claim 1. For every choice of $i, j, m, n \geq 1, H(i, j) \cap K(m, n)=\emptyset$. Consider the case where $i \leq m$, the other case being analogous. We have $H(i, j) \subseteq \operatorname{cl}(A(i)) \subseteq$ $\operatorname{cl}(A(m))$, while $K(m, n) \subseteq \operatorname{cl}(B(m))-\operatorname{cl}(A(m))$, as required.

We may write $R(X) \cap Q$ as the increasing union of closed-discrete subsets $C(n)$ of $X$. Similarly, write $L(X) \cap Q$ as the increasing union of closed-discrete subsets $D(n)$ of $X$. For each $n \geq 1$, we define

$$
R(n)=R(X) \cap(C(n) \cup H(1, n) \cup H(2, n) \cup \cdots \cup H(n, n))
$$

and

$$
L(n)=L(X) \cap(D(n) \cup K(1, n) \cup K(2, n) \cup \cdots \cup K(n, n)) .
$$

Clearly $R(n) \subseteq R(n+1)$ and $L(n) \subseteq L(n+1)$.

Claim 2. $R(X)=\bigcup\{R(n): n \geq 1\}$ and $L(X)=\bigcup\{L(n): n \geq 1\}$. We consider any $p \in R(X)$, the argument for points of $L(X)$ being analogous. If $p \in R(X) \cap Q$, then for some $n, p \in C(n) \subseteq R(n)$, so assume $p \in R(X)-Q$. Then for some $n \geq 1, p \in A(n) \subseteq \operatorname{cl}(A(n))$. We claim $p \notin \operatorname{cl}(B(n))$. For if $p \in \operatorname{cl}(B(n))$, then $p \in \operatorname{cl}(A(n)) \cap \operatorname{cl}(B(n)) \subseteq Q$, even though we have $p \in R(X)-Q$. Hence $p \in$ $\operatorname{cl}(A(n))-\operatorname{cl}(B(n))$ so that $p \in H\left(n, j_{1}\right)$ for some $j_{1}$. If $n \leq j_{1}$, then

$$
p \in H\left(n, j_{1}\right) \subset H\left(1, j_{1}\right) \cup H\left(2, j_{1}\right) \cup \cdots \cup H\left(n, j_{1}\right) \cup \cdots H\left(j_{1}, j_{1}\right)
$$

so that $p \in R\left(j_{1}\right)$. If $j_{1}<n$, then $p \in H\left(n, j_{1}\right) \subseteq H(n, n)$ so that $p \in R(n)$. In either case, $p \in \bigcup\{R(n): n \geq 1\}$, as claimed.

Claim 3. No point of $X$ is a limit point of both $R(n)$ and $L(n)$. Otherwise there is a point $p \in X$ and sequences $u_{i} \in R(n)-\{p\}, v_{j} \in L(n)-\{p\}$ of distinct points with $p=\lim \left\langle u_{i}\right\rangle=\lim \left\langle v_{j}\right\rangle$. Consider the points $u_{i} \in R(n) \subseteq C(n) \cup H(1, n) \cup$ $\cdots \cup H(n, n)$. Because the set $C(n)$ is closed and discrete, there is some $i_{1}$ such that $u_{i} \notin C(n)$ for each $i \geq i_{1}$. Then there is an integer $j_{1} \leq n$ such that infinitely many of the points $u_{i}$ belong to $H\left(j_{1}, n\right)$. Because $H\left(j_{1}, n\right)$ is closed in $X$, we have $p \in H\left(j_{1}, n\right)$. Similarly, by considering the points $v_{j} \in L(n)$, we conclude that $p \in K\left(j_{2}, n\right)$ for some $j_{2} \leq n$. But by Claim $1, H\left(j_{1}, n\right) \cap K\left(j_{2}, n\right)=\emptyset$, and that is impossible. Hence Claim 3 holds.

In light of Claim 3, no point of $\operatorname{cl}(R(n)) \cap \operatorname{cl}(L(n))$ is a limit point of both $R(n)$ and $L(n)$ so that in the notation of Theorem 3.1 $\operatorname{cl}(R(n)) \cap \operatorname{cl}(L(n)) \subseteq$ $R(n)^{i s o l} \cup L(n)^{i s o l} \subseteq R(n)^{i s o l} \cup L(n)^{i s o l} \cup E$. Hence assertion (b-2) of Theorem 3.1 holds.

Let $p \in E$. In the light of Claim 3, $p$ cannot be a limit point of both $R(n)$ and $L(n)$. Consider the case where $p$ is not a limit point of $L(n)$. Because $p \in E$ and $E \cap L(X)=\emptyset$ we know that $p \notin \operatorname{cl}(L(n))$ so that some neighborhood $M(p, n)$ has $M(p, n) \cap L(n)=\emptyset$. But then assertion (b-3) of Theorem 3.1 holds vacuously.

Lemma 3.5. Suppose $X$ is a perfect $G O$-space with a $G_{\delta}$-diagonal and that there are sets $R(n)$ and $L(n)$ with the properties described in part (b) of Theorem 3.1 . Then $X$ is quarter-stratifiable.

Proof of Lemma 3.5. Because $X$ has a $G_{\delta}$-diagonal, there is a sequence of open covers $\mathcal{U}(n)$ of $X$ by convex sets such that for each $x \in X, \bigcap\{S t(x, \mathcal{U}(n)): n \geq 1\}=$ $\{x\}$. We may assume that $\mathcal{U}(n+1)$ refines $\mathcal{U}(n)$ for each $n \geq 1$. Because $X$ is perfect, the set $I(X)$ is an $F_{\sigma}$-subset of $X$, so that we can write $I(X)=\bigcup\{I(n): n \geq 1\}$ where each $I(n)$ is a closed (and discrete) subset of $X$ and $I(n) \subseteq I(n+1)$. 
We begin by defining the sets $g(n, x)$. If $x \in I(X)$, let $g(n, x)=\{x\}$ for each $n$. If $x \in R(X) \cup L(X)$ define $\alpha(n, x)=\sup \{y \in L(n): y<x\}$ and $\beta(x, n)=\inf \{y \in$ $R(n): x<y\}$. The infima and suprema in the definition of $\alpha(n, x)$ and $\beta(n, x)$ are taken in the Dedekind completion of the ordered set $(X,<)$, so that $\alpha(n, x)$ and $\beta(n, x)$ might or might not be points of $X$. Note that $\alpha(n, x) \leq x \leq \beta(n, x)$ and we define

$$
g(n, x)=\left(\operatorname{Int}_{\mathrm{X}}([\alpha(\mathrm{n}, \mathrm{x}), \beta(\mathrm{n}, \mathrm{x})] \cap \mathrm{X}) \cap \operatorname{St}(\mathrm{x}, \mathcal{U}(\mathrm{n}))\right)-I(n) .
$$

Finally, if $x \in E(X)$ we define $g(n, x)=\emptyset$ if $x \in \bigcup\{g(n, y): y \in R(X) \cup L(X)\}$, and otherwise we let $g(n, x)=(M(n, x) \cap \operatorname{St}(x, \mathcal{U}(n)))-I(n)$.

Fix $n \geq 1$. We show that $\{g(n, x): x \in X\}$ covers $X$. Let $x \in X$. If $x \in$ $I(X)$, then $x \in g(n, x)$. Suppose $x \in R(X)$. Then $\alpha(n, x) \leq x \leq \beta(n, x)$ in the Dedekind compactification of $X$. If $x<\beta(n, x)$, then $x \in g(n, x)$, so assume that $x=\beta(n, x)$. Then $x \in \mathrm{cl}_{X}(R(n))$. We claim that $x$ is not a limit point of $L(n)$. If $x$ were a limit point of $L(n)$, then property (b) of Theorem 3.1 would force $x \in E(X) \cup R(n)^{i s o l} \cup L(n)^{i s o l}$. But we know that $x \in R(X)$ so that $x \notin E(X)$, and because $x$ is a limit point of both $R(n)$ and $L(n), x$ is not in $R(n)^{i s o l} \cup L(n)^{i s o l}$. Hence $x$ is not a limit point of $L(n)$, as asserted. Therefore, there is some $v>$ $x$ such that $[x, v) \cap L(n)=\emptyset$. We may assume that $v \in \operatorname{St}(x, \mathcal{U}(n))$. Using $x=\beta(n, x)$ we choose some point $z \in R(n) \cap(x, v)$, and we compute $\alpha(n, z)=$ $\sup \{y \in L(n): y<x\}$. Because no point of $L(n)$ lies between $x$ and $z$, we see that $\alpha(n, z) \leq x$ and that $x<z \leq \beta(n, z)$ in the Dedekind completion of $X$. Therefore $x \in \operatorname{Int}_{\mathrm{X}}([\alpha(\mathrm{n}, \mathrm{z}), \beta(\mathrm{n}, \mathrm{z})] \cap \mathrm{X})$. Because $z \in[x, v] \subseteq \operatorname{St}(x, \mathcal{U}(n))$, convexity of the members of $\mathcal{U}(n)$ forces $x \in \operatorname{St}(z, \mathcal{U}(n))$. Because $x \in R(X), x \notin I(n)$ and therefore $x \in g(n, z)$, as required. The case where $x \in L(X)$ is analogous, so consider the case where $x \in E$. If $x \in \bigcup\{g(n, y): y \in R(X) \cup L(X)\}$ there is nothing to prove, and if $x \notin \bigcup\{g(n, y): y \in R(X) \cup L(X)\}$, then $x \in g(n, x)$. Therefore $\{g(n, x): x \in X\}$ covers $X$.

Finally we show that if $x \in g\left(n, t_{n}\right)$ for each $n \geq 1$, then the sequence $\left\langle t_{n}\right\rangle$ converges to $x$. The argument has several cases. If $x \in E(X)$, then $x \in g\left(n, t_{n}\right) \subseteq$ $\operatorname{St}\left(t_{n}, \mathcal{U}(n)\right)$ forces $t_{n} \in \operatorname{St}(x, \mathcal{U}(n))$ and because $x \in E(X)$, that is enough to guarantee that $\left\langle t_{n}\right\rangle$ converges to $x$. Next consider the case where $x \in I(X)$. Then $x \in I(k)$ for some $k \geq 1$. Consider $n \geq k$. If $t_{n} \notin I(X)$, then $x \in g\left(n, t_{n}\right) \subseteq$ $X-I(n) \subseteq X-I(k)$, and that is impossible. Hence $t_{n} \in I(X)$ for each $n \geq k$, so that $x \in g\left(n, t_{n}\right)=\left\{t_{n}\right\}$, which forces $t_{n}=x$ for each $n \geq k$.

Consider the third case, where $x \in R(X)$. There is some $k$ with $x \in R(k)$. Note that $x \in g\left(n, t_{n}\right) \subseteq \operatorname{St}\left(t_{n}, \mathcal{U}(n)\right)$, so that $t_{n} \in \operatorname{St}(x, \mathcal{U}(n))$ for all $n \geq 1$. Because $x \in R(X)$ it will be enough to show that $x \leq t_{n}$ for each $n \geq k$. Suppose there is some $t_{j}<x$ where $j \geq k$. We consider several sub-cases, depending upon the nature of the point $t_{j}$. The point $t_{j}$ cannot be in $I(X)$, because then we would have $x \in g\left(j, t_{j}\right)=\left\{t_{j}\right\}$, contrary to $t_{j}<x$. Consider the case where $t_{j} \in R(X) \cup L(X)$ and compute $\beta\left(j, t_{j}\right)=\inf \left\{y \in R(j): t_{j}<y\right\}$. Because $x \in R(k) \subseteq R(j)$, we see that $\beta\left(j, t_{j}\right) \leq x$. Because $x \in R(X)$ it follows that $x \notin \operatorname{Int}_{\mathrm{X}}\left(\left[\alpha\left(\mathrm{j}, \mathrm{t}_{\mathrm{j}}\right), \beta\left(\mathrm{j}, \mathrm{t}_{\mathrm{j}}\right)\right] \cap \mathrm{X}\right)$, so that $x \notin g\left(j, t_{j}\right)$ contrary to $x \in g\left(n, t_{n}\right)$ for all $n \geq 1$. The only remaining possibility is that $t_{j} \in E(X)$, in which case $g\left(j, t_{j}\right) \neq \emptyset$ forces $g\left(j, t_{j}\right)=M\left(j, t_{j}\right) \cap$ $\operatorname{St}\left(t_{j}, \mathcal{U}(j)\right)-I(j)$. Recall that no point of $L(j) \cap M\left(j, t_{j}\right)$ can precede any point of $R(j) \cap M\left(j, t_{j}\right)$, so that because $x \in R(j) \cap g\left(j, t_{j}\right) \subseteq R(j) \cap M\left(j, t_{j}\right)$ we conclude that there is no point of $L(j)$ that lies in $M\left(j, t_{j}\right) \cap(\leftarrow, x]$. Because $M\left(j, t_{j}\right)$ is a convex open set containing both $t_{j}$ and $x$, the fact that $t_{j} \in E(X)$ yields some 
$w<t_{j}$ with $(w, x] \subset M\left(j, t_{j}\right)$. Hence no point of $L(j)$ lies in $(w, x]$. Now we compute $\alpha(j, x)=\sup \{y \in L(j): y<x\}$ and we see that $\alpha(j, x) \leq w<t_{j}$. Therefore $t_{j} \in \operatorname{Int}_{\mathrm{X}}([\alpha(\mathrm{j}, \mathrm{x}), \beta(\mathrm{j}, \mathrm{x})])$. In addition, $x \in g\left(j, t_{j}\right) \subseteq \operatorname{St}\left(t_{j}, \mathcal{U}(j)\right)$ yields $t_{j} \in \operatorname{St}(x, \mathcal{U}(j))$ and because $t_{j} \notin I(j)$ we have $t_{j} \in g(j, x)$ whence $g\left(j, t_{j}\right)=\emptyset$, contrary to $x \in g\left(j, t_{j}\right)$.

The fourth case, where $x \in L(X)$ and $x \in g\left(n, t_{n}\right)$, is analogous. Therefore we have proved that $\left\langle t_{n}\right\rangle$ converges to $x$ whenever $x \in g\left(n, t_{n}\right)$ for each $n \geq 1$ and the proof of Proposition 3.5 is complete

\section{Applications and examples}

We begin this section by applying results from Sections 2 and 3 to contrast the behavior of quarter-stratifiability in GO-spaces with the behavior of the property in general spaces. Banakh [2] proved that quarter-stratifiability is not hereditary to closed sets by proving that any Hausdorff space with a $G_{\delta}$-diagonal embeds as a closed subset of some quarter-stratifiable space. The behavior of quarterstratifiability in GO-spaces is very different, as can be seen from our next result.

Theorem 4.1. Let $X$ be a quarter-stratifiable GO-space. Then any subspace of $X$ is also quarter-stratifiable.

The proof of Theorem 4.1 needs a technical lemma that uses some special notation. Start with a GO space $X$ and a subset $Y \subseteq X$. Then $\left(Y,\left.\tau\right|_{Y},<\left.\right|_{Y \times Y}\right)$ is also a GO-space so that the set $R(Y)=\left\{p \in Y-I(Y):\left.[p, \rightarrow) \cap Y \in \tau\right|_{Y}\right\}$ is defined where $I(Y)$ is the set of relatively isolated points of $Y$. It is easy to see that $R(Y)=$ $((Y \cap R(X)) \cup P R(Y, X))-I(Y)$, where $P R(Y, X)$ is the set of "pseudo-R-points of $Y$ " and is defined by $P R(Y, X)=\left\{y \in Y:\left.[y, \rightarrow) \cap Y \in \tau\right|_{Y}\right.$ and $\left.[y, \rightarrow) \notin \tau\right\}$. With $P L(Y, X)$ defined analogously, we have $L(Y)=((L(X) \cap Y) \cup P L(Y, X))-I(Y)$.

Lemma 4.2. Suppose $(X, \tau,<)$ is any perfect GO-space and $Y \subseteq X$. Then the sets $P R(Y, X)$ and $P L(Y, X)$ are $\sigma$-closed-discrete in $X$.

Proof. Consider $P R(Y, X)$. For each $y \in P R(Y, X)$ there is a point $x_{y}<y$ such that $\left(x_{y}, y\right)$ is infinite and $\left[x_{y}, y\right) \cap Y=\emptyset$. Observe that if $y_{1}<y_{2}$ are in $P R(Y)$, then $y_{1}<x_{y_{2}}$ so that the collection $\mathcal{J}=\left\{\left(x_{y}, y\right): y \in P R(Y)\right\}$ is pairwise disjoint. The set $U=\bigcup \mathcal{J}$ is open and therefore is an $F_{\sigma}$-subset of the perfect space $X$, say $U=\bigcup\left\{F_{n}: n \geq 1\right\}$, where each $F_{n}$ is closed in $X$ and $F_{1} \subseteq F_{2} \subseteq \cdots$. Define $\mathcal{J}(n)=\left\{J \in \mathcal{J}: J \cap F_{n} \neq \emptyset\right\}$. Then each $\mathcal{J}(n)$ is locally finite in $X$ (indeed, each point of $X$ has a neighborhood meeting at most two members of $\mathcal{J}(n))$. Because $y \in \operatorname{cl}_{X}\left(\left(x_{y}, y\right)\right)$ for each $\left(x_{y}, y\right) \in \mathcal{J}$ it follows that each set $T_{n}=\left\{y \in P R(Y, X):\left(x_{y}, y\right) \in \mathcal{J}(n)\right\}$ is closed and discrete in $X$. Hence $P R(Y, X)$ is $\sigma$-closed-discrete in $X$, as claimed. By a similar argument, so is $P L(Y, X)$.

Proof of Theorem 4.1. Because $X$ is a quarter-stratifiable GO-space, $X$ is perfect and has a $G_{\delta}$-diagonal (see Proposition 2.2) and hence the same is true for the subspace $Y$. Theorem 3.1 gives us a $\sigma$-closed-discrete subset $Q$ of $X$ such that $R(X)-Q$ and $L(X)-Q$ are contained in disjoint, relative $F_{\sigma}$-subsets $A, B$ of the subspace $X-Q$. Let $Q_{1}=Y \cap(Q \cup P R(Y, X) \cup P L(Y, X))$. In light of Lemma 4.2. $Q_{1}$ is a $\sigma$-closed-discrete subset of $X$ and hence also of $Y$. Then

$$
R(Y)-Q_{1} \subseteq(R(X) \cup P R(Y, X))-Q_{1} \subseteq R(X)-Q_{1} \subseteq R(X)-Q \subset A
$$


because $P R(Y, X) \subseteq Q_{1}$. But then $R(Y)-Q_{1} \subseteq(Y \cap A)-Q_{1}$ and $A-Q_{1}$ is a relative $F_{\sigma}$-subset of $Y-Q_{1}$. Similarly, $L(Y)-Q_{1} \subseteq B-Q_{1}$, which is also a relative $F_{\sigma}$-subset of $Y-Q_{1}$. In light of Theorem 3.1, $Y$ is quarter-stratifiable.

Lemma 4.3. Suppose that $X$ is a perfect GO-space and that $X=Y \cup M$, where, in their relative topologies, $Y$ has a $G_{\delta}$-diagonal and $M$ is metrizable. Then $X$ has a $G_{\delta}$-diagonal.

Proof. Replacing $M$ by $M-Y$ if necessary, we may assume that $M \cap Y=\emptyset$. Because $M$ is a metrizable GO-space, Faber's metrization theorem [4] assures us that there is a dense subset $D$ of $M$ that is $\sigma$-discrete in $M$ and contains the set $J=\{x \in M$ : either $(\leftarrow, x] \cap M$ or $[x, \rightarrow) \cap M$ is open in $M\}$. Because $X$ is perfect, $D$ can be written as $D=\bigcup\left\{D_{n}: n \geq 1\right\}$, where $D_{n} \subseteq D_{n+1}$ and each $D_{n}$ is a closed discrete subset of $X$. Because $X$ is first-countable and collectionwise normal, there are collections $\left\{L(d, n): d \in D_{n}\right\}$ of pairwise disjoint open sets with the property that $L(d, n) \cap D_{n}=\{d\}$ and such that if $d \in D_{n}$ and $k \geq n$, the collection $\{L(d, k): k \geq n\}$ is a local base at $d$. Let $\mathcal{L}(n)$ be the family of all sets $L(d, n)$ for $d \in D_{n}$, together with the collection of convex components of the open set $X-D_{n}$. Then $\mathcal{L}(n)$ is an open cover of $X$ and it is straightforward to verify:

Claim 1. If $x \in D$, then $\bigcap\{S t(x, \mathcal{L}(n)): n \geq 1\}=\{x\}$ and if $x \in X-D$, then $\bigcap\{\operatorname{St}(x, \mathcal{L}(n)): n \geq 1\} \subseteq X-D$.

We know that $Y$ has a $G_{\delta}$-diagonal, so there is a sequence $\langle\mathcal{U}(n)\rangle$ of relatively open covers of $Y$ such that if $y \in Y$, then $\bigcap\{\operatorname{St}(y, \mathcal{U}(n)): n \geq 1\}=\{y\}$. Then there is a collection $\mathcal{V}(n)$ of open subsets of $X$ that covers $\bigcup \mathcal{U}(n)$ and has the property that $\{V \cap Y: V \in \mathcal{V}(n)\}$ refines $\mathcal{U}(n)$. Because $X$ is perfect, we may write the open set $\bigcup \mathcal{V}(n)=\bigcup\{F(n, k): k \geq 1\}$, where $F(n, k) \subseteq F(n, k+1)$ are closed sets. Let $\mathcal{H}_{Y}(n, k)$ be the set of all convex components of sets in the collection $\mathcal{V}(n) \cup\{X-F(n, k)\}$. Then $\mathcal{H}_{Y}(n, k)$ is an open cover of $X$, and if $y \in Y$, then $Y \cap \bigcap\left\{\operatorname{St}\left(y, \mathcal{H}_{Y}(n, k)\right): n, k \geq 1\right\}=\{y\}$.

Analogously, starting with the metrizable space $M$, find open covers $\mathcal{H}_{M}(n, k)$ of $X$ such that if $x \in M$, then $M \cap \bigcap\left\{\operatorname{St}\left(x, \mathcal{H}_{M}(n, k): n, k \geq 1\right\}=\{x\}\right.$.

Now define $\mathcal{H}(n, k)$ to be the collection of all convex components of members of the collection

$$
\left\{H_{1} \cap H_{2} \cap L: H_{1} \in \mathcal{H}_{Y}(n, k), H_{2} \in \mathcal{H}_{M}(n, k), L \in \mathcal{L}(n)\right\} .
$$

Then each $\mathcal{H}(n, k)$ is a convex open cover of $X$ that refines $\mathcal{H}_{Y}(n, k), \mathcal{H}_{M}(n, k)$ and $\mathcal{L}(n)$.

Claim 2. If $y \in Y$, then $\bigcap\{\operatorname{St}(y, \mathcal{H}(n, k)): n, k \geq 1\}=\{y\}$. Let $C=$ $\bigcap\{\operatorname{St}(y, \mathcal{H}(n, k)): n, k \geq 1\}$. Because $\mathcal{H}(n, k)$ refine $\mathcal{H}_{Y}(n, k)$, we already know that $C \cap Y=\{y\}$. We will show that $C \cap M=\emptyset$. For a contradiction, suppose there is a point $x \in C \cap M$. Because $C$ is convex, if $|C \cap M| \geq 3$, then $\operatorname{Int}_{\mathrm{M}}(\mathrm{C} \cap \mathrm{M}) \neq \emptyset$, so that $C \cap M$ must meet the dense subset $D$ of $M$. But from Claim 1 we know that because $y \in Y \subseteq X-M \subseteq X-D$, the set $C$ is disjoint from $D$, and that is impossible. In the case $1 \leq|C \cap M| \leq 2$, convexity of $C$ forces each point of $C \cap M$ to belong to $J$ and hence to $D$, so that once again we contradict $C \subseteq X-D$. Hence $C \cap M=\emptyset$, as required to establish Claim 2 .

Claim 3. If $x \in M$, then $\bigcap\{\operatorname{St}(x, \mathcal{H}(n, k)): n, k \geq 1\}=\{x\}$. Because $\mathcal{H}(n, k)$ refines $\mathcal{L}(n)$, Claim 1 shows that the assertion in Claim 3 is certain to hold whenever $x \in D$, so consider the case where $x \in M-D$. Once again, write $C=$ 
$\bigcap\{\operatorname{St}(x, \mathcal{H}(n, k)): n, k \geq 1\}$. We already know that $C \cap M=\{x\}$, so it is enough to show that $C \cap Y=\emptyset$. For a contradiction, suppose we can choose a point $z \in C-\{x\}$. Without loss of generality, suppose $x<z$. Then $[x, z] \subseteq C$, so that $(\leftarrow, x] \cap M=(\leftarrow, z) \cap M$, showing that $(\leftarrow, x] \cap M$ is open in $M$. Hence $x \in J \subseteq D$, so $x$ is a point of $C \cap D$. But because $x \in M-D$, Claim 1 yields $C \subseteq X-D$, and that is impossible. Hence Claim 3 holds.

Claims 2 and 3 show that the open covers $\mathcal{H}(n, k)$ for $n, k \geq 1$ are $G_{\delta}$-diagonal covers of $X$, as required in the lemma.

Remark 4.4. Note that the Alexandroff double arrow space, i.e., the lexicographic product space $A=[0,1] \times\{0,1\}$, is the union of two subspaces, each having a $G_{\delta}$-diagonal, and yet $A$ does not have a $G_{\delta}$-diagonal. Therefore, the metrizability hypothesis in Lemma 4.3 is necessary.

In Question 1.8 of [2], Banakh asks whether a $T_{3}$-space is quarter-stratifiable provided it is the union of two closed subspaces, each being quarter-stratifiable in its relative topology. In the category of GO-spaces, that question has an affirmative answer, as we show in part (a) of Proposition 4.5. In addition, Lemma 4.3 yields a quarter-stratifiable sum theorem for perfect GO-spaces that have a large metrizable part.

Proposition 4.5. Let $X$ be a GO-space. If $X$ satisfies any of the following conditions, then $X$ is quarter-stratifiable.

(a) $X=\bigcup \mathcal{F}$, where $\mathcal{F}$ is a $\sigma$-locally finite collection of closed subspaces of $X$, each of which is quarter-stratifiable;

(b) $X$ is paracompact and has a cover by open, quarter-stratifiable subspaces;

(c) $X$ is a perfect GO-space and $X=Y \cup M$, where $M$ is metrizable and $Y$ is quarter-stratifiable.

Proof. In this proof, for a set $S \subseteq X, \operatorname{cl}(S)$ will always denote the closure of $S$ in $X$. To prove (a), apply Proposition 2.2(e) to conclude that each member of $\mathcal{F}$, being a quarter-stratifiable GO-space, is perfect. But then, so is the space $X$, and therefore each member of $\mathcal{F}$ is a $G_{\delta}$-subset of $X$. Now part (3) of Banakh's Theorem 1.6 applies to complete the proof of (a).

To prove (b), let $\mathcal{G}$ be any cover of $X$ by quarter-stratifiable open subspaces. Because $X$ is paracompact, there is a locally-finite closed cover $\mathcal{F}$ of $X$ that refines $\mathcal{G}$. In light of Theorem 4.1 each member of $\mathcal{F}$ is quarter-stratifiable. Now apply assertion (a) of this proposition.

To prove (c), we first apply Lemma 4.3 to conclude that $X$ has a $G_{\delta^{-}}$-diagonal. Then, because the space $X$ is perfect and has a $G_{\delta}$-diagonal, we may use assertions (c) and (d) of Theorem 3.1. Because $Y$ is quarter-stratifiable, there is a set $Q_{Y} \subset Y$ that is $\sigma$-closed-discrete in $Y$ and has the property that $R(Y)-Q_{Y} \subseteq A$ and $L(Y)-Q_{Y} \subseteq B$, where $A$ and $B$ are disjoint relative $F_{\sigma}$-subsets of $Y=X-M$. Because $X$ is perfect, both $Q_{Y}$ and $I(Y)$ are $\sigma$-closed-discrete in $X$. Consider the subsets $R(M)$ and $I(M)$ of $M$. Because $M$ is metrizable, Faber's metrization theorem for GO-spaces [4] guarantees that both sets are $\sigma$-discrete-in-themselves and hence are $\sigma$-closed-discrete in the perfect space $X$. Let $Q=Q_{Y} \cup M \cup I(Y)$. Because $R(X) \subseteq R(Y) \cup I(Y) \cup R(M) \cup I(M)$ we have

$$
\begin{aligned}
R(X) \cap Q & \subseteq(R(Y) \cap Q) \cup I(Y) \cup R(M) \cup I(M) \\
& =\left(R(Y) \cap Q_{Y}\right) \cup I(Y) \cup R(M) \cup I(M),
\end{aligned}
$$


so that $R(X) \cap Q$ is a subset of a $\sigma$-closed-discrete subset of $X$. Hence $R(X) \cap Q$ is itself $\sigma$-closed-discrete in $X$. Similarly, $L(X) \cap Q$ is $\sigma$-closed-discrete in $X$. Consider the sets $R(X)-Q$ and $L(X)-Q$. We have

$$
\begin{aligned}
R(X)-Q & \subseteq(R(Y)-Q) \cup(I(Y)-Q) \cup(R(M)-Q) \cup(I(M)-Q) \\
& \subseteq R(Y)-Q \subseteq R(Y)-Q_{Y} \subseteq A .
\end{aligned}
$$

Therefore $R(Y)-Q \subseteq A-Q$. Note that $X-Q \subseteq Y-Q_{Y}$ so that because $A$ is a relative $F_{\sigma}$-subset of $Y-Q_{Y}$, we know that $A-Q$ is a relative $F_{\sigma}$-subset of the subspace $X-Q$. Similarly, $L(X)-Q \subseteq B-Q$ and $B-Q$ is a relative $F_{\sigma}$-subset of $X-Q$. Because $A-Q$ and $B-Q$ are disjoint, we may apply assertion (d) of Theorem 3.1 to conclude that $X$ is quarter-stratifiable.

Remark 4.6. The result in part (c) of (4.5) was pointed out by the referee. It contrasts with the best known result for quarter-stratifiable spaces in general, namely that $X$ is quarter-stratifiable if $X$ is the union of two quarter-stratifiable subspaces, one of which is a closed $G_{\delta}$-set. (See Theorem 1.6 in [2.)

Proposition 4.5 has a surprising corollary. Recall that for any GO-space $(X, \tau,<)$, there is a canonical LOTS $\left(X^{*}, \tau^{*},<^{*}\right)$ that contains $X$ as a closed subspace, where $X^{*}$ is obtained by adding a certain collection of isolated points to $X$ and where $<^{*}$ is a natural lexicographic extension of $<$ (see [6]). It is often of interest to know which topological properties of $X$ are passed on to $X^{*}$.

Corollary 4.7. For any GO-space $X$, the following are equivalent:

(a) $X^{*}$ is quarter-stratifiable;

(b) $X$ is quarter-stratifiable and $X^{*}$ is perfect;

(c) $X^{*}$ is metrizable;

(d) $X$ is metrizable.

Proof. That (a) $\Rightarrow$ (b) is in Proposition 2.2 and Theorem 4.1, above. Clearly (c) $\Rightarrow\left(\mathrm{d}\right.$ ), and it is known (see [6]) that if $X$ is metrizable, then so is $X^{*}$. Hence (d) $\Rightarrow$ (a). To complete the proof, we must show that (b) $\Rightarrow$ (c), so suppose $X^{*}$ is perfect and $X$ is quarter-stratifiable. Because $X^{*}=X \cup M$, where $M$ is a certain set of isolated points, assertion (c) of Proposition 4.5 yields that $X^{*}$ is quarterstratifiable and hence (see Lemma 2.2) has a $G_{\delta}$-diagonal. But any LOTS with a $G_{\delta}$-diagonal is metrizable. Hence $X^{*}$ satisfies (c).

Proposition 4.8. Suppose $X$ is a perfect GO-space with a $G_{\delta}$-diagonal. If $X$ satisfies any one of the following conditions, then $X$ is quarter-stratifiable:

(a) either $R(X)$ or $L(X)$ is $\sigma$-closed-discrete;

(b) there is a $\sigma$-locally-finite closed cover $\mathcal{C}$ of $X$ with the property that for each $C \in \mathcal{C}$, either $R(C)$ or $L(C)$ is $\sigma$-discrete.

Proof. To prove (a), consider the case where $L(X)$ is $\sigma$-closed-discrete in $X$. Let $Q=L(X)$ and apply Theorem 3.1 with $A=X-Q$ and $B=\emptyset$. To prove (b) note that each member of $\mathcal{C}$ is quarter-stratifiable in light of (a), and then apply Proposition 4.5.

Remark 4.9. In an earlier version of this paper, we asked whether property (b) in Proposition 4.5 characterized quarter-stratifiable GO-spaces. The referee answered that question by using the space of Example 4.10(c), below. 
Example 4.10. Three examples concerning quarter-stratifiability of perfect GOspaces having a $G_{\delta}$-diagonal:

(a) Let $A$ be a Bernstein subset of the usual space of irrationals, i.e., $A \subseteq \mathbb{P}$ and neither $A$ nor $\mathbb{P}-A$ contains an uncountable compact set. Let $X=\mathbb{R}$ and define $R(X)=\mathbb{Q}, L(X)=A$ and $E(X)=\mathbb{P}-A$. The resulting GO-space satisfies the hypotheses of Proposition 4.8 and hence is quarter-stratifiable.

(b) Let $C \subseteq \mathbb{R}$ be a Bernstein set in $\mathbb{R}$, i.e., an uncountable subset $C \subseteq \mathbb{R}$ with the property that neither $C$ nor $D=\mathbb{R}-C$ contains an uncountable compact subset of $\mathbb{R}$. Let $Y=\mathbb{R}, R(Y)=C$ and $L(Y)=D$ and let $E(Y)=I(Y)=\emptyset$. Then $Y$ is a separable (and hence perfect) GO-space with a $G_{\delta}$-diagonal, but $Y$ is not quarter-stratifiable in light of Theorem 3.1. because if $R(Y)=\bigcup\{R(n): n \geq 1\}$ and $L(Y)=\bigcup\{L(n): n \geq 1\}$, then by the Baire Category Theorem, for some $n_{0}$ either $\mathrm{cl}_{Y}\left(R\left(n_{0}\right)\right)$ or $\operatorname{cl}_{Y}\left(L\left(n_{0}\right)\right)$ would contain an interval $[a, b]$. If $[a, b] \subseteq \operatorname{cl}_{Y}\left(R\left(n_{0}\right)\right)$, then for some $n_{1}>n_{0}$ the set $\operatorname{cl}_{Y}\left(R\left(n_{1}\right)\right) \cap L\left(n_{1}\right)$ must be uncountable. Therefore, in light of part (b) of Theorem 3.1, the set $E(Y) \cup R\left(n_{1}\right)^{i s o l} \cup L\left(n_{1}\right)^{i s o l}$ is also uncountable. But that is impossible because $E(Y)=\emptyset$ and $S^{i s o l}$ is countable for each $S \subseteq Y$. The case where $[a, b] \subseteq \operatorname{cl}_{Y}\left(L\left(n_{0}\right)\right)$ is analogous. (Note that this space has all of the properties listed in Proposition 2.2.)

(c) There is a quarter-stratifiable GO-space $Z$ that does not have a $\sigma$-locallyfinite closed cover $\mathcal{C}$ with the property that for each $C \in \mathcal{C}$, either $R(C)$ or $L(C)$ is $\sigma$-closed-discrete. To construct $Z$, let $R, L$ be disjoint $\sigma$-compact subsets of the usual space $\mathbb{R}$ of real numbers with the property that for any open interval $J$ in $\mathbb{R}$, both $J \cap R$ and $J \cap L$ are uncountable. Modify the usual topology of $\mathbb{R}$ by making all points $p \in R$ have neighborhoods of the form $[p, b)$ for $p<b$, and by making all points $q \in L$ have neighborhoods of the form $(a, q]$. Apply Theorem 3.1 with $Q=\emptyset, A=R$, and $B=L$ to see that $Z$ is quarter-stratifiable. It is easy to see that $Z$ is a Baire space. Suppose that $\mathcal{C}=\bigcup\{\mathcal{C}(n): n \geq 1\}$ is any $\sigma$-locally-finite cover of $Z$ by closed subsets of $Z$. Then each $\mathcal{C}(n)$ is countable (because $Z$ is separable) and so some member $C_{0} \in \mathcal{C}$ must have interior in $Z$. Hence $C_{0} \cap R$ and $C_{0} \cap L$ are uncountable. Because $C_{0}$ is separable, $I\left(C_{0}\right)$ is countable and therefore $\left(R \cap C_{0}\right)-I\left(C_{0}\right) \subseteq R\left(C_{0}\right)$, showing that $\left.R\left(C_{0}\right)\right)$ must be uncountable. Because $C_{0}$ is separable, it follows that $R\left(C_{0}\right)$ cannot be $\sigma$-closed-discrete in $C_{0}$. Similarly, $L\left(C_{0}\right)$ cannot be $\sigma$-closed-discrete in $C_{0}$.

As a final example of the use of Theorem 3.1 we show that any GO-space constructed on a Q-set of real numbers is quarter-stratifiable. Recall that a $Q$-set is an uncountable subset $X \subseteq \mathbb{R}$ with the property that each subset of $X$ is a relative $F_{\sigma}$-subset of $X$ when $X$ carries the relative topology from $\mathbb{R}$. Q-sets exist in some models of ZFC, but not in others. We begin with a Q-set $X \subseteq \mathbb{R}$. We arbitrarily partition $X=R \cup E \cup I \cup L$ and create a GO-topology $\tau$ on the set $X$ so that $R(X)=R, E(X)=E, I(X)=I$, and $L(X)=L$. We say that $(X, \tau,<)$ is a GO-space constructed on $X$.

Corollary 4.11. If $X \subseteq \mathbb{R}$ is a $Q$-set and $(X, \tau,<)$ is any GO-space constructed on $X$, then $(X, \tau)$ is quarter-stratifiable. 
Proof. Every subset of $X$ is a relative $F_{\sigma}$-set in the topology $\rho$ that $X$ inherits from $\mathbb{R}$ and hence also in the topology $\tau$. Thus $(X, \tau)$ is perfect. Because the usual space $\mathbb{R}$ has a $G_{\delta}$-diagonal, so does $(X, \tau)$. Let $Q=\emptyset$. Then $R(X)-Q$ and $L(X)-Q$ are disjoint, relative $F_{\sigma}$-subsets of $(X, \rho)$ and hence also of $(X, \tau)$. By assertion (d) of Theorem 3.1, $X$ is quarter-stratifiable.

Remark 4.12. Even though assertion (a) of Proposition 4.8 does not characterize quarter-stratifiable GO-spaces, it does allow us to put quarter-stratifiable GOspaces into a more familiar context. Recall Faber's metrization theorem for GOspaces [4: A perfect GO-space with a $G_{\delta}$-diagonal is metrizable provided that both $R(X)$ and $L(X)$ are $\sigma$-closed discrete in $X$. If one, but not both, of $R(X)$ and $L(X)$ is $\sigma$-closed-discrete, then $X$ is quarter-stratifiable, but not metrizable.

\section{REFERENCES}

[1] Alster, K., Subparacompactness in Cartesian products of ordered spaces, Fundamenta Math. 87(1975), 7-28. MR0451210(56:9497)

[2] Banakh, T.O., (Metrically) Quarter-stratifiable spaces and their applications in the theory of separately continuous functions, Matematychni Studii 18(2002), 10-28. MR 1968755 (2004d:54023)

[3] Creede, G., Concerning semi-stratifiable spaces, Pacific J. Math. 32(1970), 47-54. MR0254799 $(40: 8006)$

[4] Faber, M.J., Metrizability in Generalized Ordered Spaces, Math. Centre Tracts 53(1974), Amsterdam. MR0418053 (54:6097)

[5] Kuratowski, K., Quelques problemes concernant les espaces metrique non-separables, Fundamenta Math. 25(1935), 534-545.

[6] Lutzer, D.J., On generalized ordered spaces, Dissertationes Mathematicae 89(1971), 1-30. MR0324668 (48:3018)

[7] Montgomery, D. Non-separable metric spaces, Fundamenta Math. 25(1935), 527-533.

[8] Rudin, W., Lebesgue's first theorem, Math. Analysis and Appl., Part B in Adv. in Math. Supplem. Studies 78, ed. by L. Nachbin, Academic Press (1981), 741-747. MR0634266 (82k:28006)

Department of Mathematics and Statistics, Texas Tech University, Lubbock, Texas 79409

E-mail address: bennett@math.ttu.edu

Department of Mathematics, College of William \& Mary, Williamsburg, Virginia 23187

E-mail address: lutzer@math.wm.edu 